\title{
NOTES ON TWO LEMMAS CONCERNING THE EPSTEIN ZETA-FUNCTION
}

\author{
by P. H. DIANANDA
}

(Received 18 February, 1964)

1. We use Cassels's notation and define $h(m, n), Q(m, n), Z_{h}(s), Z_{h}(1)-Z_{Q}(1)$ and $G(x, y)$ as in [1]. Rankin [5] proved that the Epstein zeta-function $Z_{h}(s)$ satisfies, for $s \geqq 1.035$, the

THEOREM. For $s>0, Z_{h}(s)-Z_{Q}(s) \geqq 0$ with equality if and only if $h$ is equivalent to $Q$.

Rankin then asked whether the theorem is true for all $s>1$. Cassels [1] answered this question in the affirmative and proved further that the theorem is true for all $s>0$.

In the proof of the theorem for $0<s \leqq 3$, Cassels used the following lemmas.

Lemma 1. $G_{y}(x, y)>0$ for $y \geqq \frac{3}{2}$ and $s>0$.

LEMMA 2. $\mathrm{G}_{x}(x, y)<0$ for $y \geqq \frac{3}{5}, 0<x<\frac{1}{2}$ and $0<s \leqq 3$.

Lemma 2 was proved for $1.035 \leqq s \leqq 2$ by Rankin and for $0<s \leqq 3$ by Cassels. Cassels's proof was based on Rankin's. V. O. Ennola (see [2]), pointed out that Cassels's proof needed revision. Recently Ennola [4] has obtained a revised proof which follows Cassels's closely. In $\$ 2$ we give a simpler revision of Cassels's proof, eliminating the partial summation used both in his proof and in Rankin's.

Lemma 1 was proved for $s \geqq 1$ by Rankin and for $0<s \leqq 1$ by Cassels. In $\S 3$ we simplify Cassels's proof and validate it for $0<s \leqq 3$. It is convenient to have a single such proof for this range since the lemma is used in the proof of the theorem only for this range, an elementary proof of the theorem, due to Rankin, being available for $s \geqq 3$.

The lemmas and the theorem in [1] are false for $s=0, Z_{h}(0)$ being -1 for all $h$ (see [3]). I am indebted to Dr. Cassels for pointing this out and for informing me about Ennola's proof and also about Emersleben's review [3].

In view of the above and statements of Cassels in $\$ 1$ of [1], we may rewrite the theorem as follows $†$ :

THEOREM. $\Gamma(s)\left\{Z_{h}(s)-Z_{Q}(s)\right\} \geqq 0$ for real $s \neq 0,-1,-2, \ldots$, with equality if and only if $h$ is equivalent to $Q$. Further,

$$
Z_{h}(0)=-1 \text { and } Z_{h}(s)=0 \text { for } s=-1,-2, \ldots
$$

2. To prove Lemma 2 for $0<s \leqq 3$ we first note that, as shown by Cassels in the first paragraph of $\$ 4$ of [1], it is sufficient to prove that

where

$$
\psi>0 \text { for } 0<x<\frac{1}{2}, \quad 0<\delta<\frac{1}{40} \text { and } 0<s \leqq 3 \text {, }
$$

$$
\begin{gathered}
\psi=\sum_{d \geqq 1} \sum_{f \geqq 1} d^{\frac{3}{2}-s} f^{s+\frac{1}{2}} \delta^{d f} \sin 2 \pi d f x . \\
+ \text { See the note added in proof. }
\end{gathered}
$$


Since $0<2 \pi x<\pi$, we have $\sin 2 \pi d f x \geqq-d f \sin 2 \pi x$. Hence, for $0<s \leqq 3$,

$$
\begin{aligned}
\psi & \geqq\left\{\delta-\sum_{s \geqq 2} f^{s+\frac{3}{2}} \delta^{f}-\sum_{d \geqq 2} d^{\frac{s}{2}-s} \delta^{d}-\sum_{d \geqq 2} \sum_{f \geqq 2} d^{\frac{s}{2}-s} f^{s+\frac{3}{2}} \delta^{d f}\right\} \sin 2 \pi x \\
& >\left\{\delta-\sum_{f \geqq 2} f^{\frac{2}{2}} \delta^{f}-\sum_{d \geqq 2} d^{\frac{3}{2}} \delta^{d}-\sum_{d \geqq 2} \sum_{f \geqq 2} d^{\frac{s}{2}} f^{\frac{2}{2}} \delta^{d+f}\right\} \sin 2 \pi x \\
& >\left(\delta-\Delta \delta-\frac{1}{4} \Delta \delta-\frac{1}{4} \Delta^{2} \delta^{2}\right) \sin 2 \pi x,
\end{aligned}
$$

where

$$
\Delta=\sum_{t \geqq 2} t^{\frac{9}{2} \delta^{t-1}}<\frac{2^{\frac{2}{2}} \delta}{1-\left(\frac{3}{2}\right)^{\frac{3}{2}} \delta}<\frac{2^{\frac{2}{2}}}{40-\left(\frac{3}{2}\right)^{\frac{9}{2}}}<\frac{2 \sqrt{2}}{4}<\frac{3}{4} .
$$

Thus

$$
\psi>\left(1-\frac{5}{4} \Delta-\frac{1}{4} \delta\right) \delta \sin 2 \pi x>0
$$

This completes the proof.

3. Cassels's proof of Lemma 1 for $0<s \leqq 1$ depended on the inequality

$$
\frac{1}{2} y \Gamma(s) \pi^{-s} G_{y}(x, y)=\theta(s)+\theta(1-s)+2 M>0 \text { for } y \geqq \frac{3}{2} \text { and } 0<s \leqq 1 \text {, }
$$

where

$$
\theta(s)=s y^{s} \pi^{-s} \Gamma(s) \zeta(2 s) .
$$

He deduced the above inequality from the fact that

$$
\theta(s)+\theta(1-s)>0.06 \text { and }|2 M|<0.01 \text { for } y \geqq \frac{3}{2} \text { and } 0<s \leqq 1 \text {. }
$$

On examination, the proof (of the inequality in $M$ ), given in the last paragraph of $\$ 5$ of [1], is seen to be valid for $0<s \leqq 3$ and not merely for $0<s \leqq 1$. Further, Lemma 1 is true for $0<s \leqq 3$ if

$$
\frac{1}{2} y \Gamma(s) \pi^{-s} G_{y}(x, y)=\theta(s)+\theta(1-s)+2 M>0 \quad \text { for } y \geqq \frac{3}{2} \quad \text { and } \quad 0<s \leqq 3 .
$$

Hence the lemma follows for $0<s \leqq 3$, since we can prove that

$$
\theta(s)+\theta(1-s)>0.24 \text { for } y \geqq \frac{3}{2} \text { and real } s,
$$

by using the fact that

$$
\pi^{-s} \Gamma(s) \zeta(2 s)=\frac{1}{2 s(2 s-1)}+\int_{1}^{\infty}\left(t^{-s-\frac{1}{2}}+t^{s-1}\right) \psi(t) d t
$$

where now $\psi(t)=\sum_{n=1}^{\infty} e^{-n^{2} \pi t}$. Clearly

$$
\begin{aligned}
& \theta(s)+\theta(1-s) \\
& \quad=\frac{y^{s}-y^{1-s}}{2(2 s-1)}+s y^{s} \int_{1}^{\infty} t^{-\frac{z}{t}}\left(t^{s-\frac{1}{4}}+t^{-s+\frac{1}{4}}\right) \psi(t) d t+(1-s) y^{1-s} \int_{1}^{\infty} t^{-\frac{1}{4}}\left(t^{s-\frac{1}{4}}+t^{-s+\frac{1}{4}}\right) \psi(t) d t .
\end{aligned}
$$


It is sufficient to consider $s \geqq \frac{1}{2}$, since $\theta(s)+\theta(1-s)$ is symmetrical about $s=\frac{1}{2}$. For $s \geqq \frac{1}{2}$,

$$
s \geqq|1-s|, \quad y^{s} \geqq y^{1-s}, \quad s-\frac{1}{4} \geqq\left|s-\frac{3}{4}\right| \quad \text { and } \quad t^{s-\frac{1}{d}}+t^{-s+\frac{1}{4}} \geqq t^{s-\frac{1}{t}}+t^{-s+\frac{z}{2}},
$$

clearly. Thus the two terms involving integrals, in the expression for $\theta(s)+\theta(1-s)$, have a non-negative sum. Hence

$$
\begin{aligned}
\theta(s)+\theta(1-s) & \geqq \frac{y^{s}-y^{1-s}}{2(2 s-1)}=\frac{y^{\frac{1}{2}} \sinh \left\{\left(s-\frac{1}{2}\right) \log y\right\}}{2 s-1} \\
& \geqq \frac{1}{2} y^{\frac{1}{2}} \log y \geqq \frac{\sqrt{ } 6}{4} \log \frac{3}{2}>0.24 .
\end{aligned}
$$

This completes the proof. It is easy to see that the proof can be shortened if we wish to prove the lemma for $0<s \leqq 1$ only.

Note added in proof. As stated by Cassels in $\$ 1$ of $[1], Z_{h}(s)-Z_{Q}(s)$ is regular and

$$
\pi^{-s} \Gamma(s) Z_{b}(s)=\pi^{s-1} \Gamma(1-s) Z_{h}(1-s)
$$

for all complex $s$. Consequently, $\Gamma(s)\left\{Z_{h}(s)-Z_{Q}(s)\right\}$ is regular for all complex $s$. Hence we may rewrite the theorem as follows: to $Q$.

THEOREM. $\Gamma(s)\left\{Z_{h}(s)-Z_{Q}(s)\right\} \geqq 0$ for real $s$, with equality if and only if $h$ is equivalent

\section{REFERENCES}

1. J. W. S. Cassels, On a problem of Rankin about the Epstein zeta-function, Proc. Glasgow Math. Assoc. 4 (1959), 73-80.

2. J. W. S. Cassels, Corrigendum to [1], Proc. Glasgow Math. Assoc. 6 (1963), 116.

3. O. Emersleben, Review of [1], Zbl. Math.

4. Veikko Ennola, A lemma about the Epstein zeta-function, Proc. Glasgow Math. Assoc. 6 (1964), 198-201.

5. R. A. Rankin, A minimum problem for the Epstein zeta-function, Proc. Glasgow Math. Assoc. 1 (1953), 149-158.

UNIVERSITY OF SINGAPORE 